\title{
Gradhiva
}

GRADHIV

Revue d'anthropologie et d'histoire des arts

$7 \mid 2008$

Le possédé spectaculaire

\section{Une ethnographie ratée}

Le modernisme brésilien, le département de Culture de São Paulo et la Missão de Pesquisas Folclóricas

A failed ethnography. Brazilian modernism, the Department of Culture in São Paolo and the Mission of Folkloric Research

\section{Fernando Giobellina Brumana}

Traducteur : Myriam Benarroch

\section{(2) OpenEdition}

Journals

Édition électronique

URL : http://journals.openedition.org/gradhiva/1033

DOI : 10.4000/gradhiva.1033

ISSN : 1760-849X

Éditeur

Musée du quai Branly Jacques Chirac

Édition imprimée

Date de publication : 15 mai 2008

Pagination : 70-83

ISBN : 978-2-915133-86-8

ISSN : 0764-8928

Référence électronique

Fernando Giobellina Brumana, «Une ethnographie ratée », Gradhiva [En ligne], 7 | 2008, mis en ligne le 03 décembre 2010, consulté le 21 avril 2019. URL : http://journals.openedition.org/gradhiva/1033 ;

DOI : 10.4000/gradhiva.1033 


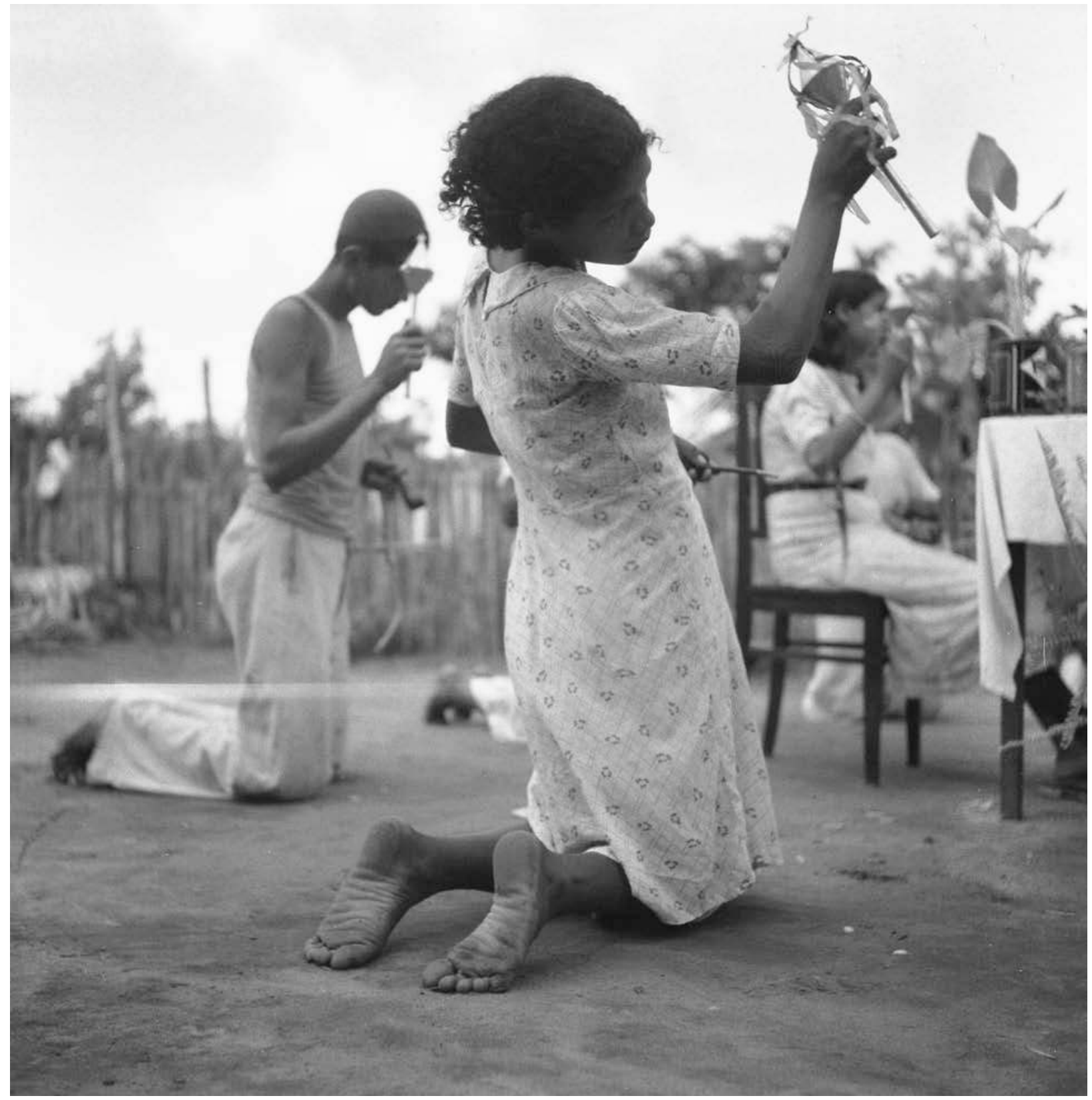

Fig. 1 Catimbó, 19 mai 1938, João Pessoa (État de Paraíba). @ Discoteca Oneyda Alvarenga, Acervo Histórico, Centro Cultural São Paulo, SMC-PMSP, photo Luis Saia. 


\title{
Une ethnographie ratée
}

\author{
Le modernisme brésilien, le département de Culture \\ de São Paulo et la Missão de Pesquisas Folclóricas*
}

\author{
Fernando Giobellina Brumana
}

$\mathrm{L}$ e modernisme brésilien se voulait une révolution esthétique et idéologique, en rupture avec « le langage pédant, artificiel et idéalisateur qui reflétait, dans la littérature "passéiste ${ }^{1 "}$ [...], la conscience idéologique de l'oligarchie rurale installée au pouvoir » (Lafetá 2000 [1974] : 21). À cette fin, comme ses équivalents européens, il pariait sur « la déformation du naturel comme facteur constructif, le populaire et le grotesque comme contrepoids au faux raffinement académiste, la quotidienneté comme refus de l'idéalisation du réel ${ }^{2}$ " (ibid. : 22). Les artistes européens en quête d'authenticité et de rénovation devaient chercher ailleurs la source de leur inspiration : l'élément archaïque autochtone, transmis par la mémoire historique, ou bien le primitif étranger véhiculé par les voyageurs et les ethnographes; au Brésil, en revanche, cette altérité à fleur de peau était inhérente à la société, y compris dans sa dimension officielle et érudite, par la présence du Noir, de l'indigène, du populaire. Le rejet de l'académisme se matérialisait donc par la revendication d'un patrimoine traditionnel (un « mariage heureux entre avant-garde et tradition » [Dassin $1978: 158]$ ).

La révolution esthétique était liée à l'appropriation d'un patrimoine artistique disséminé sur tout le territoire national, patrimoine qui, jusque-là, n'avait suscité qu'un rejet méprisant dans les hautes sphères culturel- les d'un pays, né en tant que tel à peine un siècle plus tôt, et dont les élites avaient pour projet la constitution d'une nation blanche et cultivée. La nation brésilienne de l'indépendance et, plus tard, de la République était conçue par ses créateurs et ses dirigeants comme un élan civilisateur dans un environnement naturel et racialement hostile. La nation idéale se heurtait à sa base réelle et, plus d'une fois, ces heurts furent payés par le sang, comme il advint lors de la guerre de Canudos.

Le nationalisme culturel, tel que l'envisageaient les modernistes, ne pouvait être, en termes pratiques, que le jeu et la fantaisie d'une petite élite artistique prenant place dans un marché culturel rachitique. Pourtant, en raison de circonstances exceptionnelles, cette petite élite fit un bond en avant qui lui permit de mettre en œuvre, durant une très brève période, ce qui jusque-là avait été une simple " conversation de café ». Ce fut la création du département de la Culture de la municipa-

\footnotetext{
* Cet article est une version remaniée par l'auteur et traduite de l'espagnol, de l'article intitulé « Mario de Andrade y la missão de pesquisas folclóricas (1938) : una etnografía que no fué », paru dans Revista de Indias 66 (237), 2006 : 545-572.

1. Les modernistes s'autoqualifiaient de «futuristes ».

2. Je tiens à remercier MM. José Eduardo Azevedo et Evaldo Piccino pour leur aide précieuse à la rédaction de ce texte. Ils ont, par leur important travail, permis de faire revivre la mémoire de la mission.
} 
lité de São Paulo et la nomination à sa tête de Mário de Andrade, figure la plus significative du modernisme pauliste et du Brésil tout entier. Ainsi, l'accord entre la bourgeoisie de São Paulo et les artistes et intellectuels modernistes, qui ne s'était manifesté auparavant que par un mécénat généreux et actif, devenait un fait politique, une pratique institutionnelle.

Il ne s'agissait pas d'un miracle ni d'un paradoxe. Le prêche moderniste était en phase avec les aspirations de la bourgeoisie pauliste à laquelle il offrait la possibilité, après la défaite du soulèvement constitutionnaliste de 1932, d'obtenir, par la culture, ce que n'avaient permis ni les armes ni le poids économique. Entre autres choses, il permettait de rivaliser avec la prééminence de Rio de Janeiro. La Semaine de 1922 avait déjà constitué un pas important dans cette direction. São Paulo, dont la population comportait un tiers d'étrangers, devait devenir le berceau de la nation brésilienne; d'après Paulo Duarte : « La Nation restait encore à découvrir et à inventer » (Carlini 1994 : 149). Le rôle du département, comme le stipulait son acte de constitution, était de " conquérir et [de] divulguer dans tout le pays la culture brésilienne " (Tavares Raffaini 2001 : 83); selon Patrícia Tavares Raffaini, les bandeirantes ${ }^{3}$ avaient défriché le pays, les intellectuels paulistes joueraient le même rôle en 1935.

Le national : ce qui reste lorsqu'on ôte l'élément étranger; formule simple et inquiétante. Remplacer la culture des migrants européens par une autre à construire au moyen d'investigations et de relevés folkloriques, telle était la politique du département de la Culture, qui devait être conduite en laissant de côté la majorité de la population ou en l'assujettissant. Tout compte fait, c'était une politique autoritaire et mâtinée de xénophobie. Il faut toutefois rappeler, à la décharge du personnel du département, que l'on n'opposait pas à l'élément étranger la pureté nationale, mais au contraire un métissage original, l'idée plus ou moins mythifiée des trois races : les Indiens, les Noirs et les Blancs d'origine portugaise. Les autres - Italiens, Espagnols, Polonais, Japonais, etc. -, ces groupes nationaux qui, durant les premières décennies du xxe siècle, avaient afflué massivement au Brésil, et en particulier à São Paulo, menaçaient de faire éclater une unité nationale virtuelle en une multiplicité d'îlots ignorants les uns des autres, réduisant à néant ce qui serait resté d'« authentique » dans le pays.

Le département avait assurément d'autres intentions que la revendication de ce national inventé. Elles émanaient de l'équation établie entre citoyenneté, d'un côté, éducation et culture de l'autre. Si la diffusion de la cul- ture s'effectuait sur un mode en partie traditionnel, elle impulsait aussi des contenus novateurs, un rien empreints d' « auto-exotisme »-telles les compositions musicales de Camargo Guarnieri et d'Heitor Villa-Lobos. L'équipe battait le pavé en quête d'un nouveau public, jusqu'alors insensible à la consommation de biens culturels de nature érudite. Ses moyens étaient très modestes : un ou deux jardins d'enfants, où l'on enseignait des chants venus du Nordeste, une bibliothèque itinérante - en fait un petit camion muni d'étagères, qui circulait dans les parcs fréquentés par les couches populaires pour proposer quelques livres au prêt. Le département souhaitait aussi étudier le tissu urbain de São Paulo et sa configuration afin de suggérer des modifications. Les recherches portaient sur les problèmes d'alimentation, de logement, de nettoyage de la voie publique, d'éducation; mais ce projet ne vit jamais le jour.

Le projet d'une union entre l'inspiration nationaliste et la volonté de diffusion consistait à « récupérer des éléments et des références culturelles oubliés et [à] les réinsérer dans la vie quotidienne" (ibid. 2001 : 91). La culture traditionnelle devait être réintroduite à São Paulo comme symbole de la nation que les intellectuels désiraient créer. Par exemple, la Nau Catarineta, d'origine portugaise et inconnue à São Paulo, avait été enregistrée dans le Nordeste par Guarnieri avant d'être présentée par un chœur d'enfants au Congrès national de la langue chantée; cela représentait bien peu de chose, en vérité.

Tel fut le point de départ des enquêtes sur le terrain menées par le département. Elles débutèrent par quelques incursions à l'intérieur de l'État de São Paulo, se poursuivirent par le voyage de Guarnieri à Salvador de Bahia et cessèrent brusquement lors de l'expédition de 1938. Ces trois années furent très fructueuses, surtout pour l'une des directions du département, la discothèque municipale, dirigée par la jeune Oneyda Alvarenga. « La discothèque publique fut la réalisation la plus réussie de l'un des triomphes modernistes, ce que Mário [de Andrade] appela "le droit permanent à la recherche" " (Dassin 1978 : 118). La discothèque, c'est-à-dire la musique, représentait le cadre idéal permettant la synthèse entre le populaire et l'érudit, ou plutôt la récupération du populaire par l'érudit. Voici ce qu'en dit Elizabeth Travassos:

3. Personnes qui participaient aux bandeiras. Ces expéditions, organisées aux xVII et xvIII siècles à partir du littoral de l'actuel État de São Paulo, pénétraient à l'intérieur des terres en quête de mines d'or et de pierres précieuses mais aussi d'Indiens destinés à être réduits en esclavage (NdT). 
La musique brésilienne, conçue dans «l'inconscience du peuple ", fut portée au niveau artistique par des compositeurs formés dans les écoles, dotés des meilleures techniques et pénétrés du sentiment d'un devoir historique envers la culture nationale (2000: 47).

La priorité accordée par les modernistes à la musique n'était pas chose nouvelle. Une décennie avant l'expérience du département, Andrade avait effectué plusieurs déplacements à l'intérieur du Brésil, seul ou accompagné-Blaise Cendrars fit partie du premier voyage -, qui lui permirent de recueillir un grand nombre de musiques populaires, religieuses et profanes. C'est aussi à cette période qu'il eut un contact, le seul de sa vie, avec une manifestation de la religiosité populaire, le rituel de "fermeture du corps» auquel se livraient les adeptes du catimbó. Il en fait état dans certains de ses textes.

Périphérie de Natal, périphérie de la périphérie de Natal : limites extrêmes d'un quartier misérable, où ne parviennent ni l'électricité ni le tramway. Au-delà, les dunes, la plage, la mer. «Il est impossible de décrire tout ce qui s'est passé lors de cette étrange session, mélange de sincérité et de

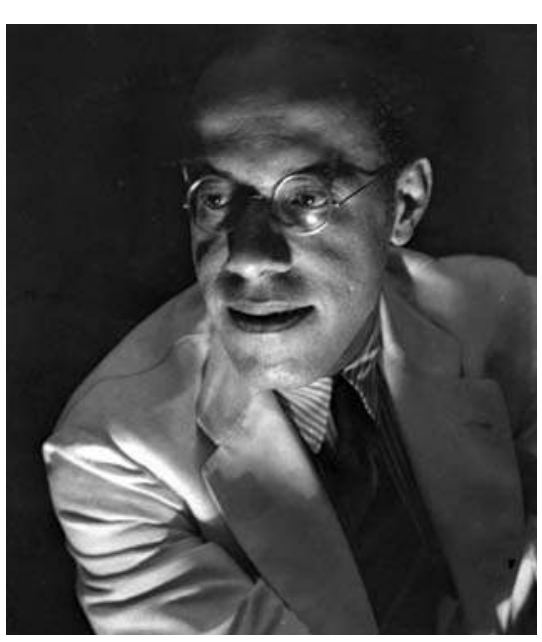

Fig. 2 Mário de Andrade. (c) Seção de Arquivo de Negativos DIM/DPH/PMSP, photo B. J. Duarte. cri sourd et rythmé par les deux "maîtres": A'iiii ! Trunfei ! Trunfá! Trunfa riá4 ! » (ibid. : 225).

Les officiants ramassèrent les pipes, les remplirent de tabac, les allumèrent aux bougies et les fumèrent à l'envers, soufflant la fumée par le tuyau sur les divers objets de la maison et sur les personnes présentes. Les prières s'unissaient aux cantiques et aux invocations. L'un des maîtres entra en transe et fit descendre en lui l'esprit d'un Indien au parler presque inintelligible et aux mouvements maladroits. L'esprit se retira pour être remplacé par un autre, qui se chargea de commencer la session et d'exécuter le travail sollicité par Andrade. D’abord, une purification : il fit tomber d'une bougie de la cire fondue sur les mains du poète; puis il se lança dans une série de gifles que le médium se flanquait à lui-même. D'autres esprits le remplacèrent alors pour poursuivre la " fermeture du corps "; enfin, arriva l'esprit le plus important, maître Carlos, qui acheva le service. «Ce fut pour moi beauté et ridicule, chants et prières, et pratiquement deux heures étranges de sensations et de diversions. Prix : 30 mille réaux " (ibid. : 227). Il s'agit, semble-t-il, de la precharlatanisme ridicule, dramatique, comique, religieux, énervant, répugnant, émouvant, tout cela à la fois. Et aussi poétique " (Andrade 2002 [1943] : 224). L'enceinte rituelle : une petite maison proprette, au sol carrelé ; pour éviter que le cri éventuel des esprits qui « descendent ", toujours imprévisibles, n'attire la police, la session se déroula au fond de l'habitation. Les deux chefs de culte qui dirigeaient le rituel firent asseoir Andrade dans l'obscurité, sur une chaise posée devant une table appuyée contre le mur. Ils allumèrent deux bougies et la scène s'illumina. Le poète était flanqué des " maîtres »-les deux chefs en personne qui, plus tard, accueilleraient en eux les "maîtres " spirituels. La table était recouverte d'une nappe blanche sur laquelle étaient posés des maracas, des pipes, de l'huile, de l'eau bénite, de l'eau-de-vie; au centre, un plat creux, la Princesse (le centre névralgique mystique du sanctuaire du catimbó). Outre les deux hommes, trois femmes assistaient à la cérémonie. Celle-ci commença par une kyrielle d'invocations à divers saints, à Dieu, aux instruments rituels, pipes et maracas. "Chaque invocation, chaque prière, était suivie d'un geste cabalistique exécuté avec les maracas, qu'accompagnait le refrain proféré dans un mière publication décrivant une cérémonie de catimbó; elle ne comportait pas d'éléments différents de celles qui paraîtraient quelques années plus tard dans les travaux de Cascudo, Gonçalves et Bastide, dont la partie ethnographique, pour être plus développée, n'était pas beaucoup plus éclairante. De plus, Andrade avait enregistré quarante-neuf cantiques du culte (paroles et partition musicale), ainsi que les paroles utilisées par ses informateurs pour lui décrire les personnages mystiques - trentetrois au total - qui apparaissaient dans les textes des chansons. Le matériel réuni par Andrade comportait également quelques oraisons et recettes de magie.

\footnotetext{
4. Voici ce que dit Mário de Andrade sur le sens de cette expression : «Le verbe trunfar était sans cesse utilisé par mes collaborateurs [c'està-dire ses informateurs]. D'après ce que j'ai pu comprendre, il s'agissait d'une contamination évidente et très naturelle entre les verbes triunfar et trunfar, vocabulaire usuel aux jeux de cartes. [...] c'est ainsi que l'importance du trunfo et du triunfo [triomphe] se conjuguaient, en un sens quelque peu confus, au sujet des dieux invoqués réticents, qui ne daignaient pas encore descendre parmi nous, les sorciers continuaient alors à chanter en rythme : A'iiii ! Trunfei ! Trunfá ! Trunfá riá » (1983 [1933] : 36). Dans le dictionnaire d'Aurélio Buarque de Holanda, trunfo - dans sa seconde acception, la première étant : «certains jeux de cartes 》- est défini comme : «carte forte qui prévaut sur les autres dans certains jeux de cartes ».
} 
$\mathrm{Au}$-delà de ses touchantes tentatives - le camionbibliothèque et les jardins d'enfants - de récupération de la culture musicale du peuple, le département de la Culture avait d'autres projets. Il finança en partie les voyages de Dina et Claude Lévi-Strauss chez les Indiens. La révolution anthropologique qui se produisit dans les années 1950 est donc liée à cette histoire.

En ce qui concerne l'ethnographie, son principal succès fut la création de la Société d'ethnologie et de folklore, au sein de laquelle Dina Lévi-Strauss dispensa un cours de préparation au travail de terrain qui donna lieu à une publication du département : «Instructions pratiques pour la recherche en anthropologie physique et culturelle " (1936). Ce texte d'une centaine de pages est consacré essentiellement à l'anthropologie physique ; seules une quinzaine de pages traitent d'ethnographie, et sur une base presque exclusivement bibliographique, car l'expérience de terrain de l'auteure se réduisait à son incursion quelques mois plus tôt chez les Kadiveu et les Bororo.

Au début, la Société d'ethnologie et de folklore était un «club». Voici ce qu'en dit une personne qui participa au projet :

On l'appelait «club», justement, pour le distinguer clairement du conventionnalisme de la Société. Il finit par tomber dans les rets du conventionnalisme et nous en avons fait une véritable Société. Mais il n'a jamais été plus vivant que lors des réunions qui se tenaient le lundi à l'école de commerce ÁlvaresPenteado, où se trouvait un musée d'ethnographie organisé, naturellement, par Dina Lévi-Strauss, qui y avait réuni plusieurs fragments de céramiques et beaucoup de vétilles (Mário Wagner Vieira da Cunha in Rubino 1995 : 504).

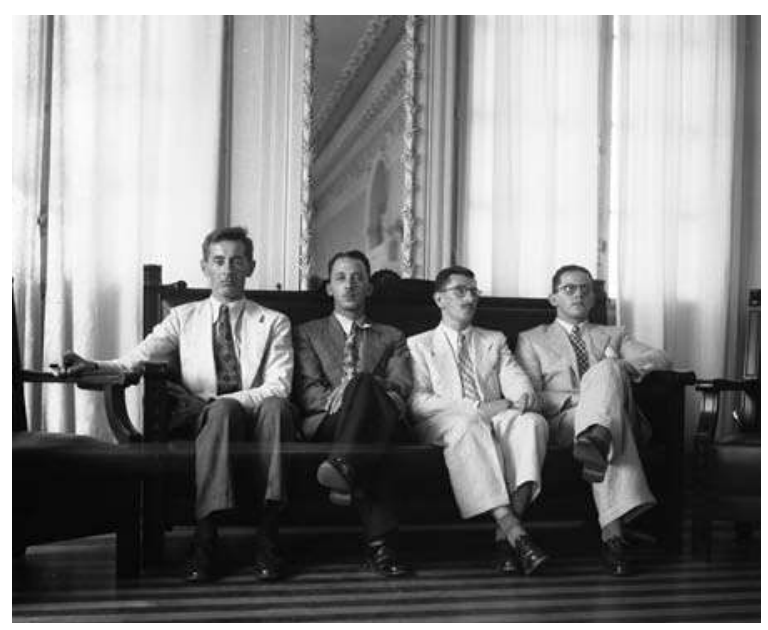

Fig. 3 Membres de la mission : Martin Braunwieser, Luis Saia, Benedicto Pacheco et Antonio Ladeira, mars 1938,

Recife (Pernambouc). $\odot$ Discoteca Oneyda Alvarenga,

Acervo Histórico, Centro Cultural São Paulo, SMC-PMSP.
Qu'il fût club ou société, ce groupe reflétait bien l'intention d'Andrade : donner une orientation scientifique au folklore, tout en construisant un cadre de collecte d'informations ethnographiques distinct d'une anthropologie culturelle et sociale pure comme des sciences naturelles. Toutefois, la rupture avec l'anthropologie physique ne faisait pas l'objet d'un consensus. Les Lévi-Strauss insistaient pour que soient prises en compte les deux disciplines, et l'une des rares recherches abouties concernait la « tache mongoloïde ", question initialement raciale que les missions françaises, rattachées à cette époque au musée du Trocadéro, incluaient dans leurs programmes au Canada, au Groenland ou en Afrique. La Société était donc hétéroclite et ne se pliait pas seulement aux désirs d'Andrade : « Le versant indigéniste de la SEF [Société d'ethnologie et de folklore] est représenté par le couple Lévi-Strauss [...] et par des traductions de textes de voyageurs [...]. L'ethnographie, pour Mário [de Andrade], c'était sa vision contemporaine du folklore » (Rubino 1995 : 503).

Des projets, le département de la Culture en avait à revendre. Certains incluaient Claude Lévi-Strauss en personne si l'on en croit Paulo Duarte, ami et protecteur d'Andrade. S'étant rendu à Paris au début des années 1930, ce dernier y avait rencontré l'état-major de ce qui devint, peu de temps après, le musée de l'Homme : Paul Rivet - auquel il consacra un livre, Rivet par lui-même-, Georges-Henri Rivière et même Marcel Mauss. Il en était revenu avec l'idée de créer un musée de la ville de São Paulo, dans le style du musée Carnavalet, avec une section historique et un département d'ethnographie qui aurait été confié à celui qui était, à ce moment-là, apprenti ethnographe ${ }^{5}$. La mission de recherches folkloriques semblait être un premier pas; elle allait, en réalité, être le dernier.

En février 1938, un groupe de quatre hommes partit de São Paulo pour répertorier les espèces culturelles menacées d'extinction par l'action corrosive de la radio et autres effets pervers de la modernité. Leur budget global (pour les déplacements, le logement, les salaires, le paiement des services et des pièces nécessaires, etc.) était de soixante millions de réaux ${ }^{6}$. Durant cinq mois,

\footnotetext{
5. Lévi-Strauss en était à ses débuts : c'était un jeune homme qui n'avait pas 30 ans, et dont on parlait d'un ton goguenard (dans une de ses lettres, Oneyda Alvarenga dit de lui : «Lévi-Strauss continue avec ses Indiens »] [cité in Peixoto 1998 : 93].

6. En dépit de diverses tentatives, je n'ai toujours pas réussi à déterminer l'équivalent actuel de cette somme. On connaît le prix de diverses choses à cette époque : nourriture pour un chanteur : onze mille réaux; paiement d'un guide : trente mille; transport de l'équipe d'enregistrement : vingt-cinq mille; location de l'éclairage : douze mille; trajet en tramway et petit-déjeuner : trois mille, etc. (Carlini 1994 : 394).
} 


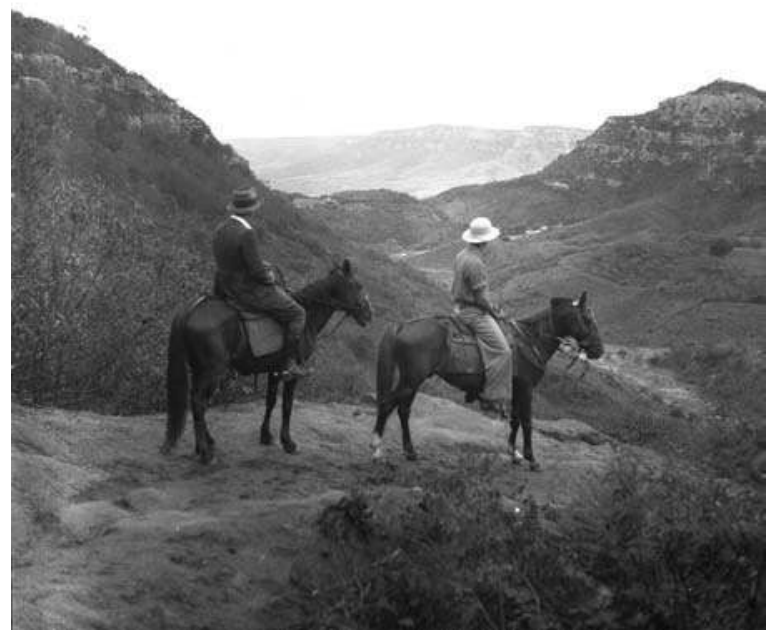

Fig. 4 Membres de la mission en route vers Brejo dos Padres (Taracatu- Pernambouc) : Antonio Ladeira et Luis Saia, mars 1938. (๔) Discoteca Oneyda Alvarenga, Acervo Histórico, Centro Cultural São Paulo, SMC-PMSP, photo Martin Braunwieser.

ils visitèrent plus de trente localités - depuis les grandes capitales comme Recife jusqu'à de petits villages inconnus (Coroatá, Cajazeiras, Corta-Dedo) - dans six États du Nordeste et du Nord (Pernambuco, Paraíba, Ceará, Piauí, Maranhão et Pará), munis d'un magnétophone Presto Record (le plus moderne de l'époque) fonctionnant avec des disques acétate, d'une caméra Kodak $16 \mathrm{~mm}$ et d'un appareil photo Rolleiflex; au total, près d'une tonne de matériel technique. Outre le chef, le jeune architecte Luís Saia (27 ans), le groupe réunissait Martin Braunwieser, musicologue autrichien, Benedito Pacheco, responsable des enregistrements, et l'adjoint Antônio Ladeira.

Ce fut certainement pour eux une grande aventure ; ils parcoururent des milliers de kilomètres en bateau, en train, en camion ou à cheval, escortés sur certains tronçons par des soldats car planait alors la menace d'une attaque par les cangaceiros ${ }^{7}$ de Lampião (Mário de Andrade avait pourtant déclaré vouloir enregistrer des chansons sur ce "bandit social ", sur le père Cícero et sur Antônio Conselheiro). L'aventure se termina brusquement : l'État Nouveau de Getúlio Vargas installa une nouvelle équipe dirigeante à la municipalité de São Paulo et, peu de temps après, Andrade dut renoncer à ses fonctions et aller travailler à Rio de Janeiro sous la tutelle du ministre de la Culture ; bien qu'ayant atteint les principaux objectifs qu'elle s'était fixés, la mission rentra avant la date initialement prévue.

Une bonne part de cette entreprise fut réalisée dans un climat politique compliqué, au point que la correspondance entre la mission et le département s'effectua dans une semi-clandestinité. Les autorités avaient leur mot à dire sur les personnes avec lesquelles Andrade et Oneyda pouvaient ou non entrer en contact. De Recife, lieu où la mission séjourna le plus longtemps, Saia écrivit à Andrade :

Parmi les secrétaires [du gouvernement de l'État de Pernambuco], celui qui a le plus de contacts avec la soutane est Manuel Luambo, avec sa clique d'extrême droite de la revue Frontières [...]. J'ai parlé avec ce membre du gouvernement. Il m'a fait tout de suite comprendre que si la mission ne voulait pas être entravée dans son travail ici, je devais m'éloigner le plus possible de Gilberto Freyre et de tous ceux qui n'appartenaient pas à la clique de Frontières. Toute désobéissance de ma part à cette injonction causerait un grand préjudice au travail de la mission, car les cartes sont entre les mains des curés (Carlini 1994: 176).

Le département se heurtait à deux problèmes particulièrement graves : sur le terrain, l'interdiction des manifestations de "bas spiritisme ${ }^{8}$ ", essentielles pour le travail envisagé par la mission ; à São Paulo, la possibilité de plus en plus grande que les nouvelles autorités municipales mettent un terme à l'expédition et de devoir, par conséquent, laisser le travail inachevé.

La situation sur le terrain n'était pas homogène. Tandis que l'État de Pernambuco avait lancé une très sévère campagne de lutte contre les manifestations de religiosité populaire, et donc contre les centres de xangô (nom attribué au candomblé dans cette région) et de catimbó, dans les autres États la répression était beaucoup moins féroce, et la mission ne connut pas de difficultés majeures pour entrer en contact avec différents groupes et enregistrer leurs cérémonies. Par ailleurs, Andrade dut demander à Saia de disparaître quelque temps dans le sertão afin qu'on ne pût le trouver et l'obliger à rentrer.

Malgré tout, les membres de la mission revinrent à São Paulo avec une belle récolte : quelque mille cinq cents mélodies répertoriées (beaucoup d'entre elles enregistrées - plus de trente heures -, d'autres simplement transcrites musicalement), six rouleaux de pellicule, des

7. Bandits armés issus de milieux ruraux défavorisés, qui circulaient en bande dans le sertão du Nordeste, à la fin du xIx et au début du $\mathrm{xx}^{\mathrm{e}}$ siècle, et vivaient de leurs attaques contre des trains, des propriétés, des petits villages ( NdT).

8. C'est la dénomination utilisée par la presse brésilienne de la fin du $\mathrm{XIX}^{\mathrm{e}}$ et du début du $\mathrm{xx} \mathrm{x}^{\mathrm{e}}$ siècle pour distinguer certaines croyances et pratiques du spiritisme kardeciste institutionnalisé, jouissant d'une légitimité certaine - sauf aux yeux de l'Église catholique. Le terme ne recouvre pas un contenu précis : il peut faire référence à tout type de manifestation médiumnique, y compris d'origine africaine. 
centaines de photographies. Venaient s'y ajouter six cents " pièces ethnographiques » de toutes sortes - instruments de musique, outils, tenues de vacher, ex-voto et, surtout, objets sacrés utilisés dans les cultes interdits -, destinées à un musée qui devait être rattaché à la discothèque et qui, comme tant d'autres projets, ne vit jamais le jour. Le succès dont témoignait cette riche moisson serait suivi d'un profond échec.

Le matériel ayant servi de support aux enregistrements, en acétate sur base d'aluminium, était très fragile, tant du point de vue de la conservation que de la qualité de l'écoute. Plus d'une fois, dans ses lettres, Alvarenga avait mis en garde Saia : il fallait éviter de passer les disques enregistrés car le matériel ne permettait qu'une seule écoute fidèle. Le manque d'intérêt des autorités pour le projet fit qu'il fallut attendre 1945, c'està-dire sept ans après la réalisation des enregistrements, pour que soient accordés à la discothèque les moyens d'exécuter des copies de ces disques, ce qui ne fut pas fait intégralement. C'est un organisme étranger, la bibliothèque du Congrès de Washington, qui publia pour la première fois une sélection des plus de trente heures d'enregistrement. La correspondance entre la directrice de la discothèque et son prédécesseur à ce poste fait état de discussions sur la politique à suivre face aux deman-

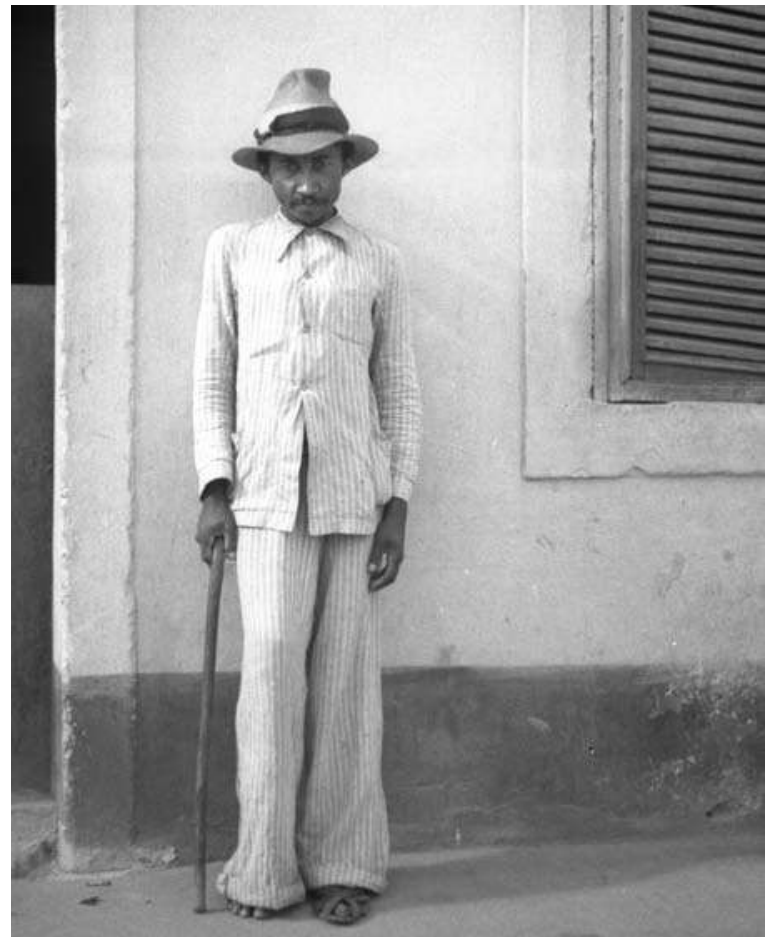

Fig. 5 Maître de catimbó, informateur Anísio José Xavier, 6 mai 1938, Alagoa Nova (État de Paraíba). @ Discoteca Oneyda Alvarenga, Acervo Histórico, Centro Cultural São Paulo, SMC-PMSP, photo Luis Saia.

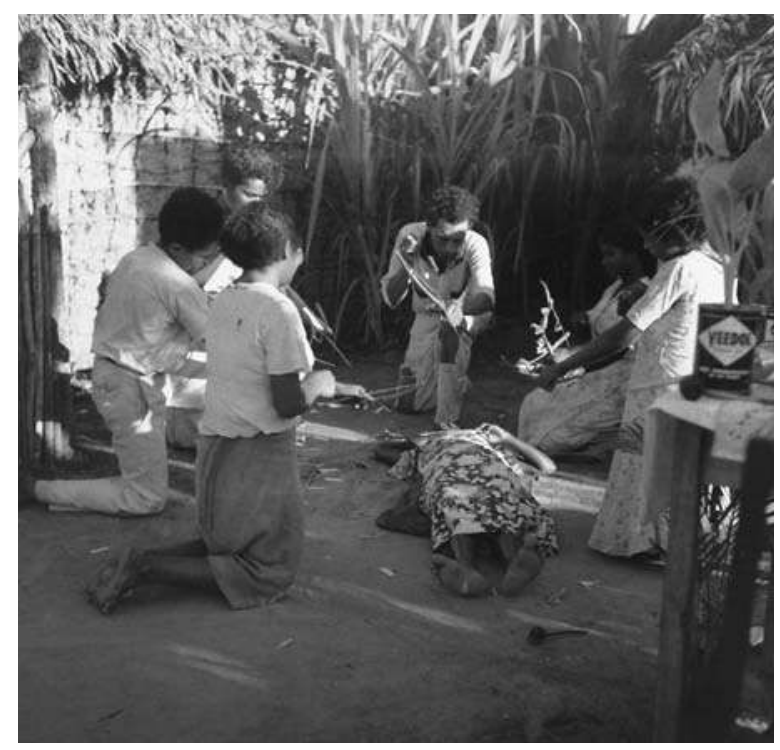

Fig 6 Catimbó, 19 mai 1938, João Pessoa (État de Paraíba). ( Discoteca Oneyda Alvarenga, Acervo Histórico, Centro Cultural São Paulo, SMC-PMSP, photo Luis Saia.

des des Américains (Andrade et Alvarenga 1983 : 221-255) et des astuces pathétiques inventées afin d'obtenir les moyens nécessaires pour enregistrer sur du matériel plus résistant les musiques proposées aux Américains : "Avec élégance et sincérité nous volerons à Spivacke [chef de la division de la Musique de la bibliothèque du Congrès] quelques disques vierges, en alléguant les dommages subis au cours des voyages, les enregistrements de copies défectueuses et autres subtilités [...]» (Mário de Andrade à Oneyda Alvarenga, 9 mai 1940, ibid.).

En 1948-1949, lorsque Alvarenga publia enfin la série de livres accompagnant les disques, sortis précédemment, elle affirma que les archives folkloriques de la discothèque publique municipale n'éditeraient rien d'autre avant longtemps. Et ce n'est qu'aujourd'hui, presque soixante-dix ans après la mission, qu'est menée à terme la tâche entreprise à l'époque. Quant aux pièces de musée, elles sont restées durant des décennies enfermées dans des cartons impossibles à localiser, rongées par les termites, rouillées ou encore perdues dans quelque trou noir de la bureaucratie ; une partie de ce matériel a été récemment présenté au centre culturel de São Paulo (2000) mais ne dispose toujours pas d'un lieu permanent d'exposition?.

Ces pièces exigent qu'on les examine à nouveau. Quelques-unes d'entre elles furent acquises par achat direct, mais de nombreuses autres, en particulier la

9. Depuis peu, des photographies et des enregistrements musicaux de la mission sont disponibles sur l'excellent site www. centrocultural. sp.gov.br. 


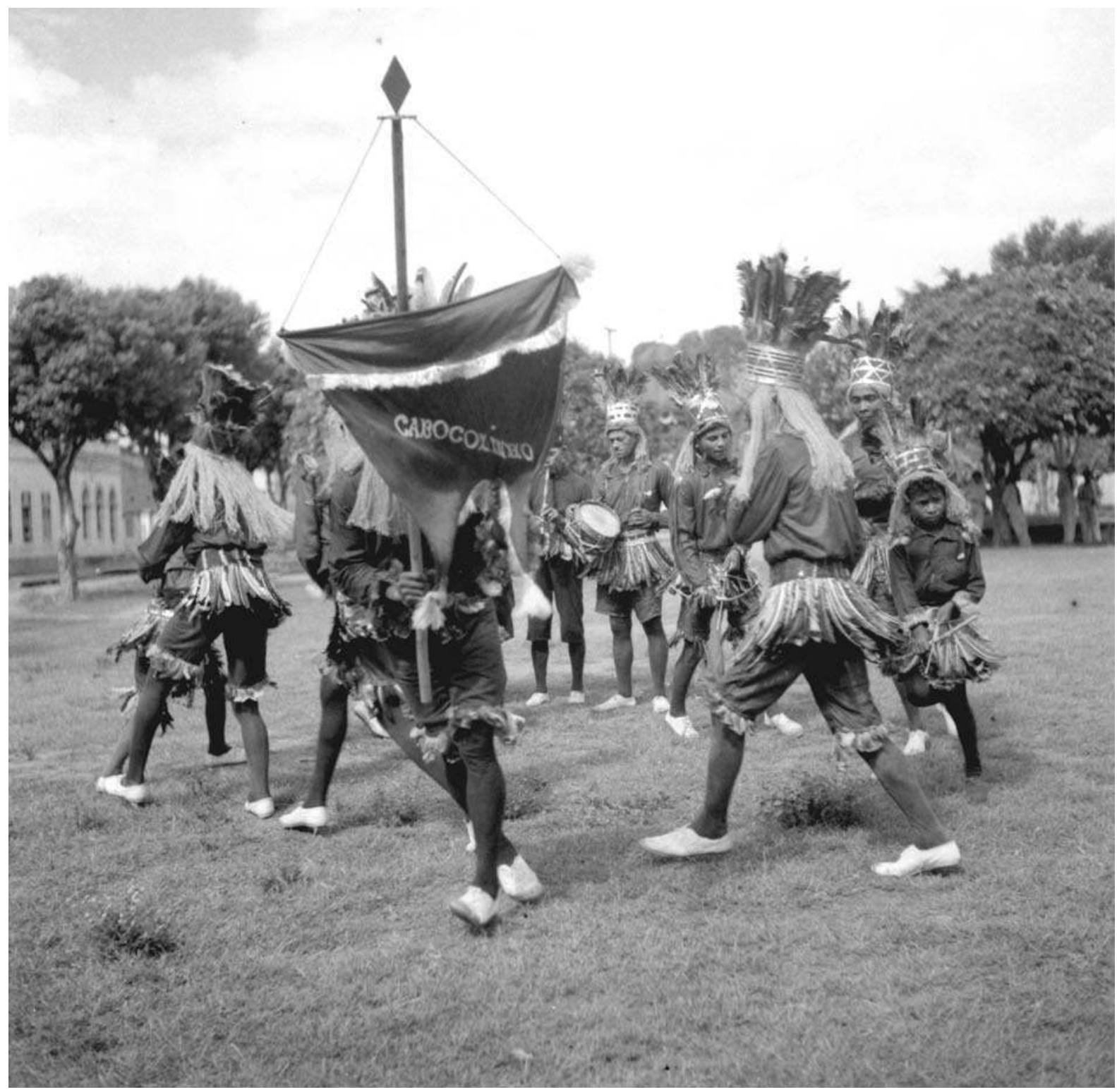

Fig. 7 Caboclinho, 4 mai 1938, Itabaiana (État de Paraíba). @ Discoteca Oneyda Alvarenga, Acervo Histórico, Centro Cultural São Paulo, SMC-PMSP, photo Luis Saia.

plupart des objets de culte, viennent d'une donation faite par la police, qui les avait réquisitionnées. Leurs propriétaires, les chefs des cultes religieux, étaient ceuxlà mêmes dont la mission avait voulu récupérer et divulguer les connaissances, tout au moins en matière musicale. La police procéda de la façon suivante : elle opéra des descentes dans plusieurs lieux de culte et s'appropria une grande quantité d'objets hétéroclites ; lorsque les gens de la mission se présentèrent, elle les chargea de classer ce matériel avec l'aide de prêtres payés pour la circonstance. En fin de compte, le chef de la police remit cet abondant matériel aux seules personnes qui s'y étaient intéressées :
Monsieur le chef de la MPF [Missão de Pesquisas Folclóricas, "Mission de recherches folkloriques "]:

Afin de faciliter la mission que vous dirigez [dans le but de recueillir] des éléments et des données sur le culte de Xangô, je prends la liberté de vous offrir les 519 pièces que vous vous êtes employé à étudier et à répertorier. Je profite de l'occasion pour vous renouveler l'expression de ma profonde estime et de ma haute considération.

José Inácio Roma (Carlini 1994 : 194).

Le butin que les membres de la mission emportèrent à São Paulo était le fruit du saccage des temples, souvent suivi de l'emprisonnement des prêtres et de la 


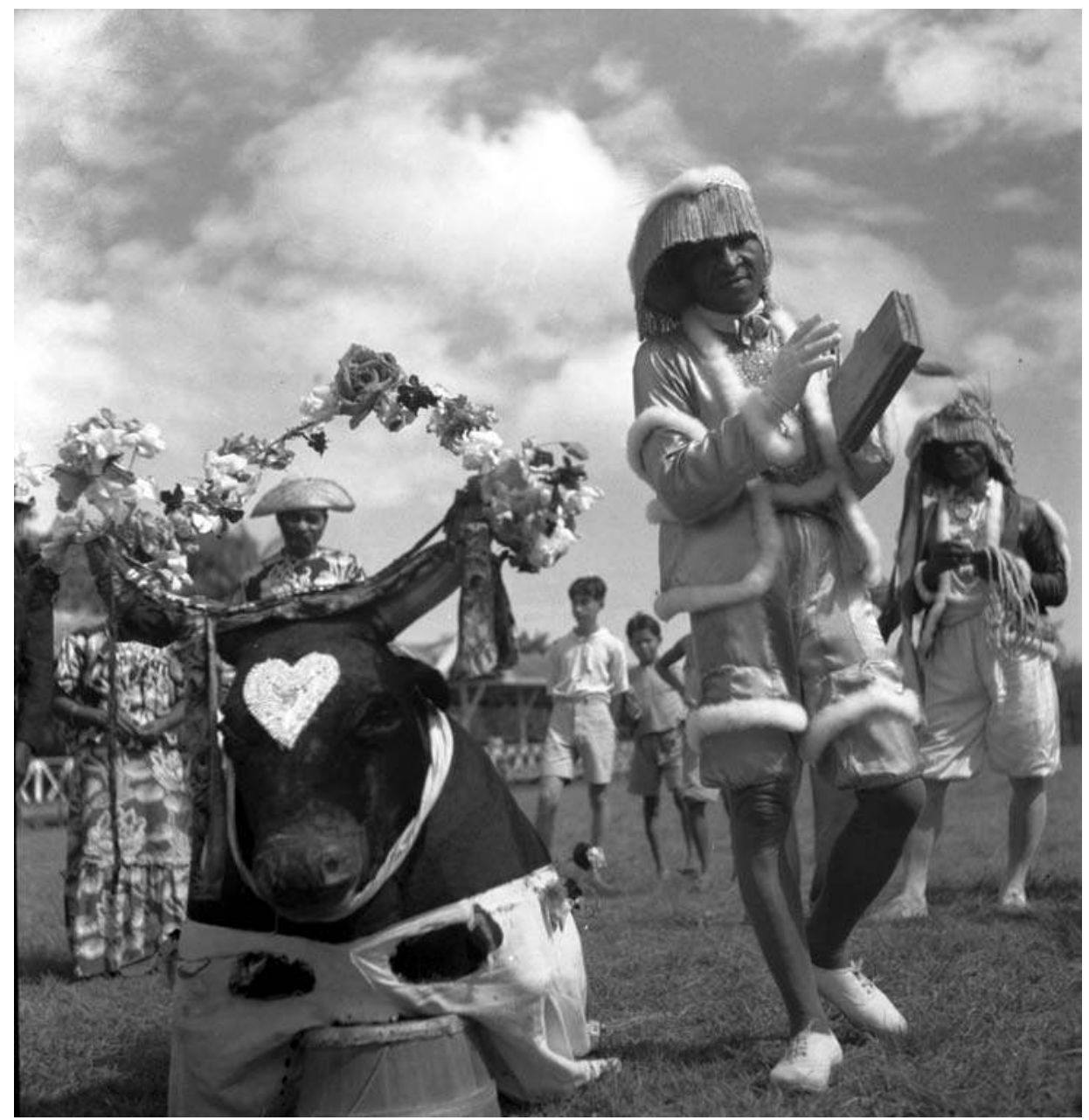

Fig. 8 Groupe de «Boi-Bumba Pai do Campo " (Père des Champs), 29 juin 1938, Belém (État du Pará). (c) Discoteca Oneyda Alvarenga, Acervo Histórico, Centro Cultural São Paulo, SMC-PMSP, photo Luis Saia.

fermeture des lieux de culte. Le fait qu'ils n'en aient pas été troublés pour autant est éloquent. Les fiches du catalogage ne mentionnent même pas la manière dont étaient disposées les pièces, en dépit des exhortations à la scientificité proférées par Andrade, qui souhaitait que l'information la plus complète sur les répertoires folkloriques soit disponible.

Andrade pas plus que ses collaborateurs ou ses détracteurs ne songèrent un seul instant à cet aspect des choses; quant aux commentateurs qui viennent après eux, ils n'abordent pratiquement pas la question. C'est dire à quel point était enracinée dans la mentalité des gens du département de la Culture la conviction que ces cultes avaient un caractère subalterne et que l'appareil d'État avait toute légitimité pour intervenir afin de protéger la population contre ceux qui exploitaient la crédulité de ses couches les plus incultes. C'est dire aussi à quel point ils n'avaient aucun doute quant au caractère non religieux, à la nature purement et simplement superstitieuse de ces pratiques. D'autre part, d'après Carlini (1994 : 219), Andrade devait offrir à ses nouveaux chefs politiques des résultats probants, et la quantité de caisses contenant les objets recueillis constituait un bon moyen de justifier le travail de la mission et, par conséquent, du département. Dans un rapport remis au maire de São Paulo (ibid. : 221), Andrade fait remarquer que la plupart de ces objets ont été acquis gratuitement.

Toujours est-il qu'aujourd'hui encore le musée de la Police militaire de Rio de Janeiro expose des objets de culte réquisitionnés durant la répression (Boyer 1996 : 7). Mais les choses ne sont pas si simples. Même ceux qui auraient dû être les plus indignés par la confiscation des objets de culte, c'est-à-dire les prêtres de ces cultes réprimés, n'eurent aucun scrupule à proposer leurs services à la mission pour aider à préparer les envois à São Paulo : 


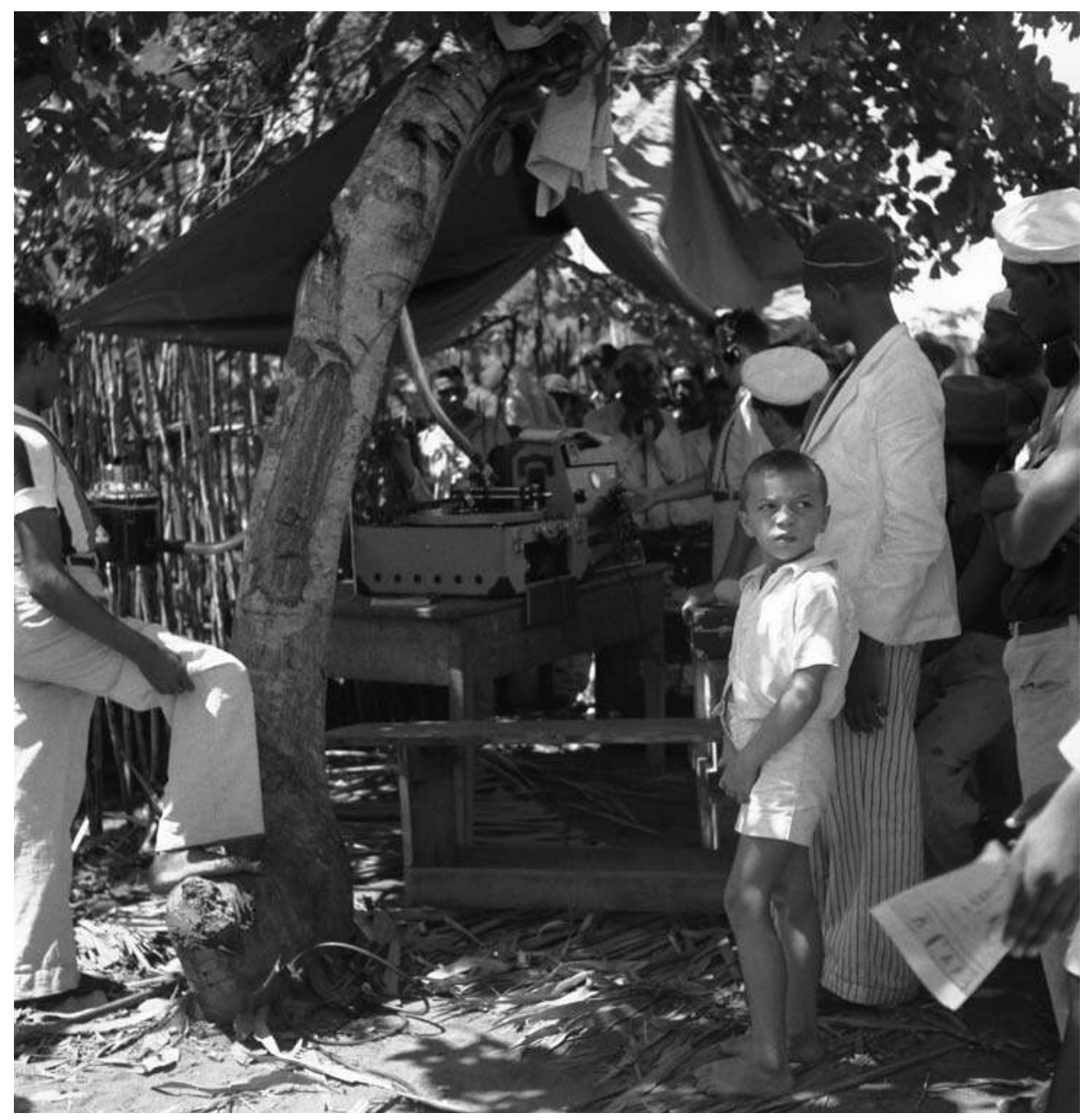

Fig. 9 Scène de collecte de matériel sonore, Torrelândia, João Pessoa (État de Paraíba). ๑ D Discoteca Oneyda Alvarenga, Acervo Histórico, Centro Cultural São Paulo, SMC-PMSP.

José Brito de Lima et le babalorixá ${ }^{10}$ Apolinário Gomes da Mota collaborèrent à l'identification des objets et fournirent divers éclaircissements sur les chants, la chorégraphie et le culte Xangô, et firent même don de documents portant sur certains aspects de la sorcellerie locale. Durant le travail d'identification des objets, la mission prit en charge tous les frais afférents (Carlini 1994 : 194).

Ce n'est pas la modique rétribution remise à ces personnes qui suffit à expliquer une telle coopération. Entre le mépris témoigné par les agents de police pour les objets de culte - condamnés de toute évidence à être détruits ou jetés à la poubelle - et l'intérêt académique des membres de la mission, le choix était vite fait. Cette collaboration s'explique sans doute aussi par le sentiment des adeptes de la religiosité populaire vis-à-vis des centres de pouvoir et d'érudition; peut-être voulaient-ils donner à ces émissaires de la grande ville du Sud la meilleure image possible d'eux-mêmes et de leur système de croyances et de pratiques. Les chefs des centres de culte avec lesquels la mission avait été en contact dans les États de Pernambuco et de Paraíba n'étaient nullement naïfs.

Au cours des enquêtes menées à propos d'autres manifestations populaires, Saia et son équipe avaient rencontré des personnes qui n'avaient jamais vu un camion de leur vie, ou qui s'étaient mises à pleurer de surprise et d'émotion en écoutant les enregistrements qu'on avait faits d'elles. Dans le cas du xangô et du catimbó, le panorama est différent : il ne s'agit pas de personnes isolées et ignorantes du monde environnant. Les informations de Saia accompagnant les enregistrements musicaux de catimbó révèlent que tous les chefs de culte ont voyagé dans plusieurs États et ont séjourné dans des villes de la dimension de Recife - où l'un d'eux

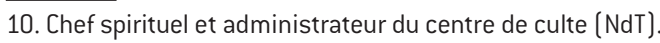


avait justement effectué son apprentissage religieux. Bien plus, l'un des maîtres de catimbó interrogés sur l'utilisation d'herbes à des fins médicales en vient à citer un travail d'érudition : l'étude sur le catimbó de Câmara Cascudo figurant dans les actes du premier congrès afro-brésilien organisé à Recife quelques années plus tôt, publiés en 1934 sous le titre Novos Estudos AfroBrasileiros. Les hommes de la mission qui s'étaient présentés à la porte de cet adepte du culte avaient l'air vivement intéressés par ce qu'il savait, croyait et faisait, mais ils donnaient aussi une impression de richesse et de pouvoir. Pour satisfaire leur curiosité, ce n'est pas à ses connaissances personnelles, acquises par transmission orale au contact de quelque maître de catimbó plus âgé et plus sage que lui, qu'il avait recours, mais au travail de recherche d'un homme appartenant à la même classe sociale qu'eux. C'était probablement pour le maître de catimbó un moyen sûr de pouvoir répondre à leurs attentes.

L'aisance avec laquelle se déplaçaient les gens de la mission leur permit d'accéder à des espaces qui auraient été interdits à d'autres, car dans ces milieux régnait une grande méfiance due à la répression exercée à cette époque contre le bas spiritisme. En dépit des difficultés, Saia réussit à nouer des liens avec des adeptes du xangô et du catimbó de Recife. Il réalisa, avec une autorisation officielle, un enregistrement de xangô au théâtre Santa Isabel, situé dans le centre-ville; pour le catimbó, il ne réussit qu'à faire une transcription graphique de quelques musiques et de leurs paroles. On peut regretter que les gens de la mission n'aient pas approfondi sur le plan ethnographique des relations si prometteuses, mais telle est la tonalité générale qui présida à la réalisation de ce travail.

Cette situation soulève une question essentielle. Le temps consacré à chacun des contacts, qu'il s'agisse de petits caboclinhos ${ }^{11}$, de porteurs de pianos, de bumbameu-boi ${ }^{12}$ ou de catimbó était dérisoire. Tout était fait à la hâte et dans une improvisation totale. Il ne fut même jamais question de partager des moments de convivialité avec le groupe qui fournissait l'information. Un exemple :

À 9 h 30, nous sommes partis à la recherche d'un catimbozeiro ${ }^{13}$ du nom de Zé Hilário, habitant à Caxangá (à cinq lieues en direction de Cariri). Nous nous sommes arrêtés à Alagoa da Roça où nous avons rencontré un handicapé qui avait été guéri par Zé Hilário et avait appris ses cantiques. Nous avons commencé à décharger le camion et [...] Pacheco s'est rendu compte que nous n'avions pas apporté de microphone. À présent, il est
3 h 10 de l'après-midi et nous sommes à Alagoa Nova où nous devons enregistrer l'handicapé, et nous n'avons pas encore déjeuné (Luís Saia, cité dans Carlini 1994 : 283).

Avant tout, l'idée même de répertorier massivement les musiques était en totale contradiction avec une ethnographie satisfaisante des groupes en question; il est évident que le choix d'une compilation extensive annulait d'avance les recherches intensives. Le caractère folklorique de la mission et la frénésie avec laquelle elle était menée expliquent les faibles résultats obtenus du point de vue ethnologique. À détacher les musiques de leur contexte et de ce qui leur donnait du sens, à vouloir enregistrer tous les airs et toutes les paroles des chansons, l'équipe se priva de la possibilité de produire une ethnographie religieuse - et pas seulement religieuse dotée d'un minimum de rigueur et de pertinence. Ce qu'Andrade, Saia et Alvarenga appelaient « ethnographie » avait pour seul objectif de «fournir les données nécessaires au contrôle de la validité scientifique des enregistrements, des textes des mélodies et des documents afférents » (Alvarenga 1949 : 6). L'« information ethnographique » accompagnant les enregistrements des catimbós est réduite à un petit questionnaire soumis à la personne interviewée : nom, race, âge, filiation, travail, degré d'alphabétisation, déplacements, lieux d'acquisition du savoir sur le culte. Rien de plus. Un exemple :

Luís Gonzaga Ângelo, inf. n²77. Mulâtre. 34 ans. Né à Goiana, État de Pernambouc. Parents : Francisco Vieiga Ângelo et Maria Filomena Ângelo, nés tous deux dans le Paraíba. Sachant lire et écrire. Exerce la profession de forgeron. A voyagé dans les États de Rio de Janeiro, Minas Gerais, Bahia et à Vitória. C'est le maître de ce catimbó enregistré à João Pessoa, et le soliste de tous les phonogrammes répertoriés (ibid. : 19).

Parfois, l'information est tout simplement infondée : les cinq membres d'un catimbó enregistré par la mission déclarent n'avoir jamais assisté auparavant à une session. C'est Alvarenga elle-même qui dénonce de manière très précise la piètre qualité de l'« ethnographie » de la mission.

11. «Groupes déguisés en Indiens, avec leurs fifres et leurs petites flûtes, qui parcourent les rues les jours de carnaval. Ils exécutent une danse primaire, au rythme du son des flèches frappées sur les arcs, mimant l'attaque et la défense et de simples troca-pés [mouvements que produit le corps selon les appuis du pied : à droite, à gauche, devant et derrière] » (Cascudo 1955).

12. D'après le dictionnaire de langue portugaise au Brésil Aurélio: «Danse populaire tragicomique, organisée en un cortège de personnages humains, animaliers et fantastiques, dont les péripéties tournent autour du thème de la mort et de la résurrection du bœuf. »

13. Pratiquant du catimbó (NdT). 
Comme dans les autres collectes d'informations qu'elle a réalisées, la MPF n'a pas organisé ni expliqué ses observations sur les catimbós. Le matériel informatif qu'elle a rapporté se réduit, dans sa presque totalité, à quelques notes prises rapidement par Luís Saia et dispersées dans divers carnets. Saia n'a que rarement dactylographié les données recueillies, essentiellement des textes de cantiques, mais il l'a fait sans aucun caractère systématique et sans éclaircissements plus précis (ibid. : 10).

Dans l'ouvrage dédié à la musique de catimbó, Alvarenga signale parfois les faiblesses des données ethnographiques : "[...] le matériel informatif écrit rapporté par la MPF est inconsistant », "On ne peut pas savoir [...] », «[...] est noté de manière peu claire[...] ", «[...] n'est pas expliqué [...] », « Luís Saia s'est lui aussi trompé $[\ldots]$ ", «... du fait de l'imprécision des recherches [...] », «[...] l'indigence des rapports... ».

Si l'information recueillie par la mission est un peu plus riche sur le catimbó que sur le xangô et les différents cultes amazoniens, elle est aussi plus décevante.

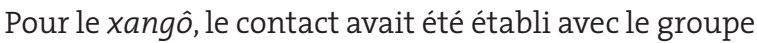
qui avait réalisé les enregistrements au théâtre Santa Isabel et, plus tard, avec les prêtres qui avaient aidé à classifier les objets de culte offerts par la police. La tâche laborieuse de récupération et d'organisation des don- nées réalisée par Alvarenga à partir du matériel dispersé de Saia aboutit à la publication du premier volume des «Registres sonores de folklore musical brésilien ", Xangô, consacré à la présentation des musiques et paroles de ce culte recueillies par la mission à Recife. Le matériau éparpillé dans les notes de Saia a servi à contextualiser les enregistrements. Il s'agit de données personnelles obtenues grâce à un questionnaire semblable à celui employé pour les membres du catimbó et portant, cette fois-ci, sur dix-sept hommes et femmes, distingués par leur condition de solistes (six) ou de choristes (onze); d'une liste sommaire du panthéon des saints avec leurs correspondants catholiques et quelques légendes; de la traduction des cantiques en « africain ». Pour le babassué, le tambor de Mina et le tambor de crioulo - cultes d'origine africaine caractéristiques de certaines régions du nord du Brésil -, la mission ne réalisa que des enregistrements musicaux et chorégraphiques; les notes de Saia ne nous indiquent même pas de quelle façon les chercheurs ont accédé aux groupes de culte dont ils ont enregistré les cérémonies.

Les éléments de catimbó recueillis font état de trois groupes de culte de l'État de Paraíba, l'un dans la capitale, les deux autres dans de petites localités de la

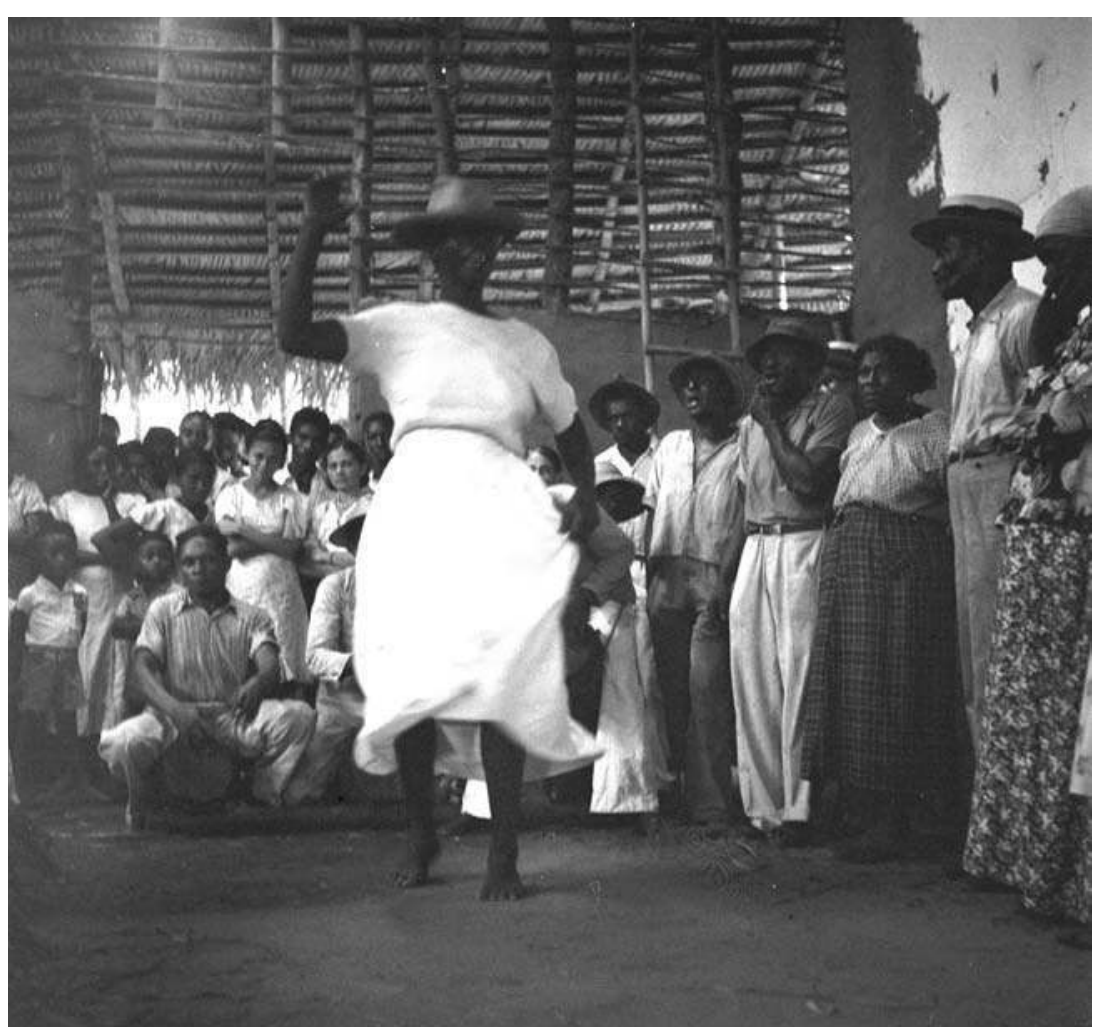

Fig. 10 Tambor de crioula, 19 juin 1938, São Luis do Maranhão. @ Discoteca Oneyda Alvarenga, Acervo Histórico, Centro Cultural São Paulo, SMC-PMSP, photo Luis Saia. 
région. Viennent s'ajouter des informations sur un catimbó de Recife tirées d'entretiens avec le chef du groupe réalisés par Saia. Ces matériaux ont été eux aussi récupérés et organisés par Alvarenga dans son troisième volume des "Registres sonores du folklore musical brésilien ", sur la base de diverses fiches et de carnets annotés par Saia. En dehors de l'aspect strictement musical et chorégraphique, traité avec grande précision et rassemblant de nombreux détails, il s'agit de notes quasi télégraphiques qui cachent plus qu'elles ne révèlent, ou plutôt qui révèlent à quel point il y avait là une information très riche qu'on n'a pas voulu, su ou pu recueillir.

Prenons pour exemple une imprécision et un malentendu autour de l'appellation du culte pratiqué par Luís Gonzaga Ângelo, mentionné plus haut. Alors que les membres de la mission parlaient de catimbó, les acteurs et natifs employaient le terme de xangô. Soulignons que le mot catimbó revêt, tout au moins depuis quelques décennies, un caractère péjoratif, considéré comme un synonyme de "sorcellerie ». Aucune recherche n'a été menée sur ses contacts avec les maîtres de catimbó qui lui ont enseigné les airs repris par son groupe. L'histoire religieuse de l'informateur reste ainsi dans le flou; nous ne saurons rien de ce qui a motivé son adhésion au culte, des mécanismes de son initiation, des raisons qui l'ont poussé à " ouvrir son propre lieu de culte », de la manière dont il a recruté les autres membres du groupe, etc.

On nous propose une longue liste de " plantes utilisées dans les travaux ", où sont indiqués le mode d'administration (bains, ingestion, fumigation), les affections concernées, ainsi que des recettes plus complexes. Dans cet inventaire figurent des remèdes populaires « empiriques » aux effets mystiques. De quoi parle-t-on? Existe$\mathrm{t}$-il des liens emblématiques entre espèces végétales et entités spirituelles ? Y a-t-il des précautions à prendre, des normes mystiques à suivre pour recueillir, préparer et utiliser les herbes en question?

Le lien avec le spiritisme auquel Saia fait parfois référence n'est pas exploré ; de même, on n'explique pas l'ancrage temporel du catimbó, qui existe à Recife dès les années 1880 comme en témoigne l'indication ponctuelle sur l'histoire religieuse fournie par un autre catimbozeiro, Manuel Laurentino (Alvarenga 1949 : 79). Rien n'est dit de la structure des groupes de culte ni de leur dynamique ; la pratique simultanée du catholicisme est tout juste mentionnée sans qu'on sache quels sont les biens et services de la religion dominante consommés par les adeptes du catimbó, etc.

Toutes ces critiques doivent toutefois être tempé- rées, comme nous l'avons fait plus haut en rappelant les conditions politiques dans lesquelles s'est effectuée la mission, l'inexpérience de ses membres, l'urgence de la tâche entreprise. Que serait-il advenu si la mission avait disposé d'une seconde chance, si les critiques émises par Alvarenga sur son déroulement avaient pu conduire vers de nouvelles recherches?

L'équipe constituée par Mário de Andrade n'a pas survécu à son auteur et timonier ; elle s'est désagrégée, ou plus exactement éparpillée. Saia s'engagea dans un autre organisme officiel, Braunwieser retourna à l'enseignement, on perdit la piste de Pacheco et de Ladeira durant des décennies, les Lévi-Strauss quittèrent le Brésil, la Société d'ethnologie et de folklore disparut. La convergence entre une ethnographie d'avant-garde et le souci de «brésilianité », esquissée avec plus ou moins de bonheur dans l'expérience de la mission et dans le projet du département de la Culture, fut gelée durant plusieurs décennies. Le souvenir même de la mission s'était effacé jusqu'à une date récente.

Le projet d'Andrade fut laminé par le Brésil officiel et par Wenceslao Pietri Petra, l'ennemi juré de Macunaíma ${ }^{14}$ au sein de l'État. Englué dans l'inertie étriquée de la machine bureaucratique, Pietri Petra voyait dans un pari comme celui du département de la Culture plus qu'un danger, pire qu'un danger : une perte de temps et d'argent. On dit que l'exil d'Andrade à Rio après son départ du département finit par entraîner sa mort prématurée. Il écrivit à un ami :

On m'accuse de trop boire, mais tant que je me trouve dans cette petite ville, comment retrouver le rythme d'antan, réveils matinaux, quelle idée! C'était une époque antédiluvienne où l'on parlait de l'existence d'un département qui eut la stupidité d'être culturel dans cette [bourgade de] Loanda [São Paulo].

mots clés / keywords : Brésil // Brazil • modernisme // modernism • religiosité populaire // popular religiosity • histoire de l'ethnologie // history of ethnology.

Université de Cadix fernando.giobellina@uca.es

14. Antihéros du roman homonyme de Mário de Andrade, sans doute la plus importante de ses œuvres, publiée en 1928.

Traduction de l'espagnol par Myriam Benarroch. 


\section{Bibliographie}

\section{Alvarenga, Oneyda}

1948 Xangô. São Paulo, Discoteca Pública Municipal.

1949 Catimbó. São Paulo, Discoteca Pública Municipal.

\section{AndRADE, Mário de}

1983 [1933] Música de feitiçaria no Brasil. Belo Horizonte, Itataia.

2002 [1943] 0 turista aprendiz.

Belo Horizonte, Itataia.

Andrade, Mário de

et Alvarenga, Oneyda

1983 Cartas. São Paulo, Livraria Duas Cidades.

BARBATO Jr. et ARRUDA, Roberto

Luiz de

2001 Missionários de uma Utopia nacionalpopular: os intelectuais e o departamento de Cultura de São Paulo, thèse de doctorat. Universidade de Campinas.

BOyer, Véronique

1996 « Le don et l'initiation. De l'impact de la littérature sur les cultes de possession au Brésil », L'Homme 138.

\section{CÂMara CAscudo, Luís da}

1973 Dicionário do Folclore Brasileiro. Rio de Janeiro, Ouro.

1988 [1934] « Notas sobre o catimbó », Novos Estudos Afro-Brasileiros. Recife, Massangana.

\section{CARLINI, Álvaro}

1993 Cachimbo e maracá: o Catimbó da Missão (1938). São Paulo, Centro Cultural de São Paulo.

1994 Cante lá que gravam cá, mémoire de maîtrise. São Paulo, USP-Centro Cultural de São Paulo.

\section{Centro cultural de São Paulo}

2000 Acervo de Pesquisas Folclóricas de Mario de Andrade 1935-1938. São Paulo, Prefeitura de São Paulo.

\section{DAssin, Joan Rosalie}

1978 Política e poesia em Mario de Andrade. São Paulo, Livraria Duas Cidades.

\section{LAFETÁ, João Luiz}

2000 [1974] 1930: A crítica e o Modernismo.

São Paulo, Livraria Duas Cidades.

\section{PeIXoto, Fernanda Arêas}

1998 «Lévi-Strauss no Brasil: a formação do antropólogo », Mana. Estudos de Antropologia Social 1, vol. IV : 79-108.

\section{RuBino, Silvana}

1995 «Clube de Pesquisadores. A Sociedade de Etnografia e Folclore e a Sociedade de Sociologia », in Sérgio MISCELI (dir.), Historia das Ciencias Sociais no Brasil, vol. II. São Paulo, Sumaré.

\section{TAVARES RAFFAINI, Patrícia}

2001 Esculpindo a Cultura na Forma Brasil. o Departamento de Cultura de São Paulo [1935-38]. São Paulo, Humanitas-FFLCH/USP.

\section{TRaVAssos, Elizabeth}

2000 Modernismo e música brasileira. Rio de Janeiro, Jorge Zahar.

\section{Résumé / Abstract}

Fernando Giobellina Brumana, Une ethnographie ratée. Le modernisme brésilien, le département de Culture de São Paulo et la Missão de Pesquisas Folclóricas. - Cet article porte sur le projet inspiré et développé par le modernisme brésilien afin de recenser le patrimoine musical, d'usage fondamentalement religieux, du Nord et du Nordeste brésilien. Concrètement, il présente la tentative menée à la fin des années 1930 de constituer une archive d'ethnomusicologie, mission financée et dirigée par le département de la Culture de la ville de São Paolo. Mário de Andrade (l'auteur de Macunaíma) dirigea pendant environ deux ans ce département, institution au sein de laquelle Claude Lévi-Strauss a fait ses premiers pas en tant qu'ethnologue.
Fernando Giobellina Brumana, A failed ethnography. Brazilian modernism, the Department of Culture in São Paolo and the Mission of Folkloric Research. - The subject of this article is the project inspired and developed by Brazilian modernism, in order to preserve the musical heritage, used for fundamentally religious purposes, of Northern and Northeastern Brazil. It is substantially concerned with the attempt carried out at the end of the 1930s to create an ethnomusicological archive, which was financed and directed by the Department of Culture of the City of São Paolo. Mario de Andrade (the author of Macunaíma) directed this secretariat for about two years, and it was in this institution that Lévi-Strauss took his first steps as an ethnologist. 